\title{
Developing a Survey for Analyzing Attitudes of Adolescent and Young Adult Cancer Survivors Towards Diet, Exercise, and Social Media
}

\author{
Samantha Rodriguez¹, Tammy Sajdyk, PhD, $\mathrm{MS}^{2}$, Jamie Renbarger, MD, $\mathrm{MS}^{2}$ \\ ${ }^{1}$ Indiana University School of Medicine; ${ }^{2}$ Indiana University School of Medicine, Department of \\ Pediatrics
}

Background and Hypothesis: Due to improved multidrug treatments over the past 40 years, the 5-year survival rate for children diagnosed with cancer has increased from approximately $60 \%$ to $85 \%$. However, these treatments also cause long-term health issues, so patients must be equipped with adequate information regarding potential future complications. Only $18 \%$ of survivors report follow-up visits to a physician to discuss reducing risks resulting from their cancer, thus, there is a huge gap in health education for this population. To address this gap, a questionnaire was developed to collect data on the critical concerns adolescent and young adult (AYA) survivors have regarding wellness. We hypothesize that this survey will allow for healthcare workers to have a greater understanding of the wellness needs of AYA cancer survivors and aid in the development of an educational program that can be delivered in a manner that is acceptable to the AYA survivor population.

Project Methods: A 5-point Likert scale survey was created to gather information on topics such as diet, exercise, body image, relationships, and social media. Additional questions that gather data on background information were also asked. This survey will be distributed to subjects who have recovered from cancer and are between the ages of 18 and 39. After data has been collected, statistical tests such as Spearman's correlation and chi-square tests for independence will be used to find correlations.

Expected Results: Results from this survey will be useful in understanding the experiences of AYA cancer survivors, how they view their wellness needs, and how they prefer to receive healthcare information.

Potential Impact: The data from this study will inform oncology providers as to which health concerns the AYA survivor population find most important and how best to deliver that information so survivors can receive better management of their long-term health. 